\title{
Genotypic and phenotypic features of all Spanish patients with McArdle disease: a 2016 update
}

\author{
Alfredo Santalla ${ }^{1,2+}$, Gisela Nogales-Gadea ${ }^{3,5^{*}+}$, Alberto Blázquez Encinar ${ }^{2,5,6}$, Irene Vieitez ${ }^{7}$, \\ Adrian González-Quintana 2,5, Pablo Serrano-Lorenzo 2,5 , Inés García Consuegra 2,5,6, Sara Asensio 2,5, \\ Alfonsina Ballester-Lopez ${ }^{3,5}$, Guillem Pintos-Morell ${ }^{3,4,5}$, Jaume Coll-Cant1 $1^{3,5,8}$, Helios Pareja-Galeano ${ }^{2,9}$, \\ Jorge Díez-Bermejo ${ }^{2,9}$, Margarita Pérez ${ }^{9}$, Antoni L. Andreu ${ }^{10}$, Tomàs Pinós ${ }^{5,10}$, Joaquín Arenas ${ }^{2,5}$, \\ Miguel A. Martín ${ }^{2,5,6}$ and Alejandro Lucia 2,9
}

From 34th FIMS World Sports Medicine Congress

Ljubljana, Slovenia. $29^{\text {th }}$ September $-2^{\text {nd }}$ October 2016

\begin{abstract}
Background: We recently described the genotype/phenotype features of all Spanish patients diagnosed with McArdle disease as of January 2011 ( $n=239$, prevalence of 1/167,000) (J Neurol Neurosurg Psychiatry 2012;83:322-8). Several caveats were however identified suggesting that the prevalence of the disease is actually higher.

Methods: We have now updated main genotype/phenotype data, as well as potential associations within/between them, of all Spanish individuals currently diagnosed with McArdle disease (December 2016).

Results: Ninety-four new patients (all Caucasian) have been diagnosed, yielding a prevalence of 1/139,543 individuals. Around 55\% of the mutated alleles have the commonest PYGM pathogenic mutation p.R50X, whereas p.W798R and p. G205S account for 10 and 9\% of the allelic variants, respectively. Seven new mutations were identified: p.H35R, p.R70C, p. R94Q, p.L132WfsX163, p.Q176P, p.R576Q, and c.244-3_244-2CA. Almost all patients show exercise intolerance, the second wind phenomenon and high serum creatine kinase activity. There is, however, heterogeneity in clinical severity, with $8 \%$ of patients being asymptomatic during normal daily life, and $21 \%$ showing limitations during daily activities and fixed muscle weakness. A major remaining challenge is one of diagnosis, which is often delayed until the third decade of life in $72 \%$ of new patients despite the vast majority (86\%) reporting symptoms before 20 years. An important development is the growing proportion of those reporting a 4-year improvement in disease severity (now 34\%) and following an active lifestyle (50\%). Physically active patients are more likely to report an improvement after a 4-year period in the clinical course of the disease than their inactive peers (odds ratio: 13.98; 95\% confidence interval: 5.6, 34.9; $p<0.001$ ). Peak oxygen uptake is also higher in the former ( $20.7 \pm 6.0$ vs. $16.8 \pm 5.3 \mathrm{~mL} / \mathrm{kg} / \mathrm{min}, p=0.0013)$. Finally, there is no association between PYGM genotype and phenotype manifestation of the disease.

(Continued on next page)
\end{abstract}

\footnotetext{
*Correspondence: gnogales@igtp.cat

${ }^{\dagger}$ Equal contributors

${ }^{3}$ Grup de Recerca en Malalties Neuromusculars i Neuropediatriques,

Department of Neurosciences, Institut d'Investigació en Ciències de la Salut

Germans Trias i Pujol, Universitat Autònoma de Barcelona, Camí de les

Escoles, s/n 08916 (Barcelona), Badalona, Spain

${ }^{5}$ Centre for Biomedical Network Research on Rare Diseases (CIBERER),

Instituto de Salud Carlos III, Madrid, Spain

Full list of author information is available at the end of the article
} 
(Continued from previous page)

Conclusions: The reported prevalence of McArdle disease grows exponentially despite frequent, long delays in genetic diagnosis, suggesting that many patients remain undiagnosed. Until a genetic cure is available (which is not predicted in the near future), current epidemiologic data support that adoption of an active lifestyle is the best medicine for these patients.

Keywords: McArdle disease, Spanish patients, Genotype, Phenotype, Glycogenosis type V

\section{Background}

Glycogenosis type $\mathrm{V}$ [glycogen storage disease type $\mathrm{V}$ (GSD V), McArdle disease or myophosphorylase deficiency; $\left.\mathrm{OMIM}^{\circ} 232,600\right]$ is an autosomal recessive disease of carbohydrate metabolism. It is caused by inherited pathogenic mutations in the gene Phosphorylase, Glycogen, Muscle (PYGM), encoding the muscle-specific isoform of glycogen phosphorylase, myophosphorylase, which catalyzes the breakdown of glycogen into glucose-1phosphate in this tissue [1]. This myopathy is arguably the prototype of exercise intolerance, which typically consists of acute crises of early fatigue and contractures, occasionally accompanied by rhabdomyolysis and myoglobinuria [1]. The current best diagnostic tool for McArdle disease is genetic testing to determine whether patients are homozygous or alternatively compound heterozygous for pathogenic PYGM mutations [1]. Yet the so-called 'second wind' phenomenon, that is, marked improvement in tolerance to dynamic exercise (eg, bicycling at a constant, submaximal wattage) after 6-10 min of exertion, with subsequent disappearance of previous tachycardia, is a unique characteristic of patients with McArdle disease that is easily measurable [2]. Thus, laboratory assessment of this second wind can be used to support (or discard) the presence of McArdle disease before eventual genetic diagnostic confirmation.

Epidemiologic data available on McArdle disease are relatively scarce and usually limited in sample size [3-7]. We recently described the main genotype and phenotype features of all Spanish patients diagnosed with McArdle disease, as of January 2011 [8]. According to our prior study, reporting on the largest series of McArdle patients published to date $(n=239)$, the prevalence of the disease was $\sim 1 / 167,000$ Spanish individuals of Caucasian descent. Several caveats were however identified that led us to believe that the actual prevalence of the disease might be higher [8]. A number of patients are likely to remain undiagnosed owing to the rarity of the disease (which is still not well known by many clinicians) or to the mildness of the symptoms in some cases (with no actual interference with daily living activities). Further, many paediatricians are probably unaware of the fact that McArdle disease is to be considered, to a large extent, a paediatric condition, which should expedite diagnosis. In fact, only $4 \%$ of Spanish patients were genetically diagnosed during the first decade of life (despite $58 \%$ of the total reporting onset of symptoms during childhood), and $47 \%$ had not been diagnosed until 30+ years of age [8]. Our previous observations concur with those of recent population estimates by De Castro and colleagues using next-generation sequencing (NGS) of the PYGM gene [9]. These authors suggested that the currently accepted prevalence of McArdle disease in Americans of European descendent ( 1/ $100,000)[10]$ is an underestimate, with the actual disease prevalence being at least 2 -fold higher, and thus $\geq 3$-fold higher than the prevalence we recently reported for Spanish patients [8].

The diagnostic protocol followed by the National Health System, where the blood of candidate patients is routinely sent to each of 3 'reference' centres for genetic analysis (Hospital 12 de Octubre, Madrid; Hospital Val d'Hebron, Barcelona; and Hospital Meixoeiro, Vigo), makes it relatively easy to gather data on Spanish McArdle patients. Further, an increasing number of patients with exercise intolerance are referred to our exercise physiology facilities (Universidad Europea de Madrid or Universidad Pablo Olavide, Seville), which allows us to assess the second wind. Thus, the aim of this study was to update the main genotype and phenotype characteristics, as well as potential associations within/between them, of all Spanish individuals who are currently diagnosed with McArdle disease.

\section{Methods}

We have used a cross-sectional design to perform an update of the main PYGM genotype and phenotype data [clinical and laboratory variables (muscle biopsy when available, serum creatine kinase (CK) activity), exercise capacity] of all Spanish individuals diagnosed with McArdle disease, as of December 7th, 2016. The study also has a prospective element, as we have followed-up the 4-year progression of the clinical severity of the disease (see below) in a sub-cohort of 151 patients (ie, $n=89$ from 2006 to 2010, already reported by us [8], and $n=62$ from 2011 and onwards). The study was approved by the local ethics committees and followed the tenets of the Declaration of Helsinki, 1961.

\section{PYGM genotyping}

Mutant PYGM alleles were identified in patients' blood samples using SNaPShot mini-sequencing (ThermoFisher) or polymerase chain reaction and restriction fragment 
length polymorphism methods [11], followed by: Sanger sequencing of the entire coding region and intron/exon boundaries of the PYGM gene [12], or the use of a NGS customised gene panel on a PGM-IonTorrent platform (ThermoFisher), consisting of 35 genes, including PYGM, associated with metabolic myopathies. In some cases, analysis of muscle or blood mRNA/cDNA was needed to demonstrate the molecular pathogenicity of a presumed mutant allele, particularly when an alteration of the splicing mechanism was suspected $[13,14]$.

\section{Phenotype data}

We recorded from clinical histories [or from personal interview with (and direct evaluation of) the patients (in those visiting the aforementioned exercise physiology laboratories for functional evaluation)] data on comorbidities, exercise intolerance, self-reported second wind phenomenon, permanent muscle weakness [15, 16], basal serum CK activity after 1+ days with no exercise (last result available), muscle biopsy results (corroborating lack of staining for myophosphorylase and no myophosphorylase activity in biochemical analyses), and clinical severity class following the classification originally reported by Martinuzzi et al. [5].

Since autumn 2010, we (AS, AL, MP) have interviewed 151 patients on the progression of their disease within the previous 4-year period to ascertain: (i) improvement (change to a lower severity class in the aforementioned Martinuzzi's scale), (ii) worsening (change to a higher severity class), or (iii) constant (no change). On the same day of the interview, patients were also asked about their physical activity (PA) habits and were classified as physically active if they followed international PA guidelines, that is, doing $\geq 150 \mathrm{~min} /$ week of moderate-vigorous PA (walking/brisk walking, bicycling, swimming) $[17,18]$.

Patient's peak oxygen uptake $\left(\mathrm{VO}_{2}\right.$ peak $)$ was determined during cycle-ergometry [19] or treadmill testing until exhaustion (in children) as reported elsewhere [19], and the second wind diagnostic test was performed following the methodology reported by Vissing and Haller [2] using consistently the same equipment (metabolic cart and cycle-ergometer) and under the supervision of the same researchers (AS, AL, MP).

\section{Statistical analysis}

Descriptive data are expressed as frequencies (\%) and mean \pm standard deviation. We compared phenotype data between genders with the $\chi^{2}$ test or unpaired Student's $t$ test. To determine whether the clinical condition of patients deteriorates with aging, we compared patients' age between the different severity groups with 1-factor analysis of variance (and with Tukey's test for post hoc comparisons), and between patients showing an improvement in disease progression vs. those showing worsening/ no change (with unpaired Student's $t$ test).
We also calculated the odds ratio (OR) and 95\% confidence interval $(\mathrm{CI})$ to determine the association: between PYGM genotype and disease phenotype/progression, on the one hand, and between PA levels and disease progression, on the other. All statistical analyses were performed using the PASW (v.18.0 for WINDOWS, Chicago) and the level of significance was set at 0.05 .

\section{Results}

Compared with the first report on all diagnosed Spanish patients as of January 2011 [8], 94 new patients of Spanish nationality (all of Caucasian descent) have been diagnosed with McArdle disease in only 5 years, to sum a total of 333 patients (183 male). Three patients (all males) included in the previous report [8] have died since January 2011, all due to cardiovascular disease, at the age of 56, 67 and 89 years. As such, the prevalence of McArdle disease in the Spanish (Caucasian) population is now $~ 1 / 139,543$ persons living.

Table 1 shows the PYGM mutational spectrum of Spanish patients, which in essence has not changed in the last years. The genetic analysis showed that $\sim 55 \%$ of the mutated alleles harbour the commonest Caucasian stop codon mutation p.R50X, 10\% the missense p.W798R mutation (a virtually Spanish private mutation) and $9 \%$ correspond to the p.G205S (a relatively common Caucasian mutation). The PYGM exons containing more mutations are, in decreasing order, exons $1,18,17,15$ and 12 . No mutations were found in exons 6-8. Yet, 7 novel PYGM mutations were identified: 5 missense mutations, p.H35R, p.R70C, p.R94Q, p.Q176P, p.R576Q, 1 frameshift predicting a premature stop codon (p.L132WfsX163, and 1 splice-site microdeletion mutation c.244-3_244-2CA (Additional files 1 and 2). Of note, all the patients carrying a new mutation exhibited the second wind phenomenon, as assessed by us in the laboratory, and consequently the functional diagnosis of McArdle disease was also proven in these cases. We identified the 2 mutant PYGM alleles in all but 3 people $(99.1 \%$ of total). Although only 1 mutant allele has been identified in these 3 individuals, we also consider them to be patients with McArdle disease because they also experienced the second wind phenomenon in laboratory assessment. The main reason for not having yet detected the second pathogenic mutation in these patients is simply lack of time because diagnosis analyses started only 2 months ago (October 2016).

Phenotype data, and how they compare to our previous report [8], are shown in Table 2. They do not essentially differ between sexes (data not shown, $p>0.1$ for most comparisons). The mean age of the cohort has now slightly increased since the first report (by 3 years), simply reflecting the aging of the previous 239 patients, who still account for the majority of the cohort. The main clinical features of McArdle disease have remained essentially unchanged with regard to our previous report, except for a slight decrease 
Table 1 PYGM mutations identified in all Spanish McArdle patients $(N=333)$

\begin{tabular}{|c|c|c|}
\hline Type of mutation & N & $\%$ \\
\hline p.R50X (c.148C > T) / p.R50X (c.148C > T) & 114 & $34.2 \%$ \\
\hline p.R50X (c.148C > T) / p.W798R (c.2392 T > C) & 29 & $8.7 \%$ \\
\hline p.G205S (c.613G > A) / p.G205S (c.613G > A) & 20 & $6.0 \%$ \\
\hline p.W798R (c.2392 T > C) / p.W798R (c.2392 T > C) & 16 & $4.8 \%$ \\
\hline p.R50X (c.148C > T) / p.G205S (c.613G > A) & 14 & $4.2 \%$ \\
\hline p.R50X (c.148C > T) / p.K754fsX49 (c.2262delA) & 8 & $2.4 \%$ \\
\hline p.C784X (c.2352C > A) / p.R94W (c.280C > T) & 5 & $1.5 \%$ \\
\hline p.R50X (c.148C > T) / p.R94W (c.280C > T) & 6 & $1.8 \%$ \\
\hline p.R50X (c.148C > T) / p.R602W (c.1804C > T) & 3 & $0.9 \%$ \\
\hline p.R50X (c.148C > T) / p.A660D (c.1979C > A) & 3 & $0.9 \%$ \\
\hline p.R50X (c.148C > T) / p.E383K (c.1147G > A) & 3 & $0.9 \%$ \\
\hline p.G205S (c.613G > A) / c.1768 + 1G > A & 2 & $0.6 \%$ \\
\hline p.R50X (c.148C > T) / c.1768 + 1G > A & 4 & $1.2 \%$ \\
\hline p.R50X (c.148C > T) / p.A365V (c.1094C > T) & 3 & $0.9 \%$ \\
\hline p.R50X (c.148C > T) / p.A55GfsX21 (c.163_167delGCTCT) & 2 & $0.6 \%$ \\
\hline p.R50X (c.148C > T) / p.A704V (c.2111C > T) & 4 & $1.2 \%$ \\
\hline p.R50X (c.148C > T) / p.D534fsX5 (c.1601delA) & 2 & $0.6 \%$ \\
\hline p.R50X (c.148C > T) / p.L5VfsX22 (c.13_14delCT) & 3 & $0.9 \%$ \\
\hline p.R50X (c.148C > T) / p.R194W (c.580C > T) & 2 & $0.6 \%$ \\
\hline p.R50X (c.148C > T) / p.R715W (c.2143C > T) & 3 & $0.9 \%$ \\
\hline $\begin{array}{l}\text { p.R50X (c.148C > T) / p.T488 N }(c .1463 C>A) \\
+ \text { p.K215 K (c.645G > A) }\end{array}$ & 2 & $0.6 \%$ \\
\hline p.R576X (c.1726C > T) / p.G136AfsX159 (c.407G > A) & 2 & $0.6 \%$ \\
\hline $\begin{array}{l}\text { p.R771PfsX33 (c.2310_2311dupCC) / p.R771PfsX33 } \\
\text { (c.2310_2311dupCC) }\end{array}$ & 2 & $0.6 \%$ \\
\hline $\begin{array}{l}\text { p.W388SfsX34 (c.1162_1169delTGGCCGGTinsA)/ } \\
\text { p.K754fsX49 (c.2262delA) }\end{array}$ & 2 & $0.6 \%$ \\
\hline p.W798R (c.2392 T > C) / p.K215 K (c.645G > A) & 2 & $0.6 \%$ \\
\hline p.K609 K (c.1827 G > A) / p.K609 K (c.1827 G > A) & 1 & $0.3 \%$ \\
\hline p. Y733X (c.2199C > G) + p.Y733X (c.2199C > G) & 1 & $0.3 \%$ \\
\hline $\begin{array}{l}\text { p.A55GffX21 (c.163_167delGCTCT) / p.A55GffX21 } \\
\text { (c.163_167delGCTCT) }\end{array}$ & 1 & $0.3 \%$ \\
\hline p.A660D (c.1979C > A) / p.A660D (c.1979C > A) & 1 & $0.3 \%$ \\
\hline p.E125X (c.373G > T) / p.E125X (c.373G > T) & 1 & $0.3 \%$ \\
\hline p.G174D (C.521G > A) / p.K609 K (c.1287G > A) & 1 & $0.3 \%$ \\
\hline p.G205S (c.613G > A) / p.A365V (c.1094C > T) & 1 & $0.3 \%$ \\
\hline p.G205S (c.613G > A) / p.183F (c.247A > T) & 1 & $0.3 \%$ \\
\hline p.G205S (c.613G > A) / p.Q176_M177insVQ (c.529-8 g > a) & 1 & $0.3 \%$ \\
\hline p.183F (c.247A > T)/ p.R94W (c.280C > T) & 1 & $0.3 \%$ \\
\hline p.K754NfsX49 (c.2262delA) / c.2380-1G > A & 1 & $0.3 \%$ \\
\hline p.K754NfsX49 (c.2262delA) / p.K754NfsX49 (c.2262delA) & 3 & $0.9 \%$ \\
\hline p.L116P (c.347 T > C) / p.L116P (c.347 T > C) & 1 & $0.3 \%$ \\
\hline p.L587P (c.1760 T > C) / p.A660D (c.1730A > G) & 2 & $0.6 \%$ \\
\hline p.L5VfsX22 (c.13_14delCT) / p.K754fsx49 (c.2262delA) & 1 & $0.3 \%$ \\
\hline
\end{tabular}

Table 1 PYGM mutations identified in all Spanish McArdle patients $(N=333)$ (Continued)

\begin{tabular}{lll}
\hline Type of mutation & N & $\%$ \\
\hline p.M442 K (c.1325 T > A) / p.M442 K (c.1325 T > A) & 1 & $0.3 \%$ \\
p.N134KfsX161 (c.402delC) / p.R491AfsX7 (c.1470dupG) & 1 & $0.3 \%$ \\
P.0577R (c1730A > G) / p.A660D (c.1730A > G) & 1 & $0.3 \%$
\end{tabular}

p.R194W (c.580C > T) + p.E797VfsX18 (c.2385_2386delAA)/ $1 \quad 0.3 \%$ p.R194W (c.580C > T) + p.E797VfsX18 (c.2385_2386delAA)

p.R50X $(\mathrm{c} .148 \mathrm{C}>\mathrm{T}) /{ }^{\mathrm{a}} \quad 4 \quad 1.2 \%$

p.R50X (c.148C > T) / c.(1969+214)_(2177 + 369)de $\quad 1 \quad 0.3 \%$

p.R50X (c.148C > T) / C.1827 G > A $\quad 1 \quad 0.3 \%$

p.R50X (c.148C > T) / c.855 + 5G > A $\quad 1 \quad 0.3 \%$

p.R50X (c.148C > T) / p.E349K (c.1045G > A) $10.3 \%$

p.R50X (c.148C > T) / p.G455R (c.1363G > C) $\quad 1 \quad 0.3 \%$

p.R50X (c.148C > T) / p.G695R (c.2083G > A) $20.6 \%$

p.R50X (c.148C > T) / p.K215 K (c.645G > A) $1 \quad 0.3 \%$

p.R50X (c.148C > T) / p.L587P (c.1760 T > C) $1 \quad 0.3 \%$

p.R50X (c.148C > T) / p.L5VfsX22 (c.13_14delCT) $20.6 \%$

+ p.R324G

p.R50X (c.148C > T) / p.N685Y (c.2053A > T) $110.3 \%$

p.R50X $(c .148 \mathrm{C}>\mathrm{T}) / \mathrm{p} . \mathrm{Q} 734 \mathrm{Hfs} \mathrm{X7} \quad 1 \quad 0.3 \%$

(c.211_217dupCGCAGCA)

p.R50X (c.148C > T) / p.Q755X (c.2263C > T) $110.3 \%$

p.R50X (c.148C > T) / p.R576X (c.1726C > T) $110.3 \%$

p.R50X $($ c.148C > T) / p.T488 N (c.1463C > A $) \quad 1 \quad 0.3 \%$

p.R50X (c.148C > T) / p.T692KfsX30 $1 \quad 0.3 \%$

(c.2075_2076delCCinsAAA)

p.R50X (c.148C > T) / p.W388SfsX34 $10.3 \%$

(c.1162_1169delTGGCCGGTinsA)

p.R50X (c.148C > T) / R715W (c.2143C > T) $110.3 \%$

p.R50X (c.148C > T) / p.V456 M (c.1366G > A) $110.3 \%$

p.R50X (c.148C > T) / p.L354P (c.1061 T > C) $1 \quad 0.3 \%$

p.W798R (c.2392 T > C) / p.R590H (c.1769G > A) $\quad 1 \quad 0.3 \%$

p.Y574X (c.1722 T > G) / p.K609 K (c.1827G > A) $1 \quad 0.3 \%$

p.L5VfsX22 (c.13_14delCT) / p.L5VfsX22 (c.13_14delCT) $4 \quad 1.2 \%$

p.R50X (c.148C > T) / p.E27AfsX50 (c.78_79delTG) $20.6 \%$

p.R50X (c.148C > T) / p.L116P (c.347 T > C) $110.3 \%$

c.1092-1G > T / c.2444-3_244-2delCA $110.3 \%$

p.R50X (c.148C > T) / p.K609 K (c.1287G > A) $1 \quad 0.3 \%$

p.R491Afs (c.1470dupG) / a $\quad 1 \quad 0.3 \%$

p.W388SfsX421 (c.1162_1169delTGGCCGGT)/ $110.3 \%$

p.W388SfsX421 (c.1162_1169delTGGCCGGT)

p.G205S (c.613G > A) / p.R590H (c.1769G > A) $1 \quad 0.3 \%$

p.W798R (c.2392 T > C) / ${ }^{\text {a }} \quad 1 \quad 0.3 \%$

p.R50X (c.148C > T) / p.R490W (c.1468C > T) $110.3 \%$

p.R50X (c.148C > T) / p.V456 M (c.1366G > A) $10.3 \%$

p.W798R (c.2392 T > C) / c.212_218dup (p.Q73HfsX) $1 \quad 0.3 \%$

c.2262delA (p.K754Nfs) / c.244-3_244-2delCA $110.3 \%$ 
Table 1 PYGM mutations identified in all Spanish McArdle patients $(N=333)$ (Continued)

\begin{tabular}{lll}
\hline Type of mutation & N & $\%$ \\
\hline p.K754fsX49 (c.2262delA) / c.773-2A > T & 1 & $0.3 \%$ \\
p.Q734HfsX7 (c.211_217dupCGCAGCA) / p.Q734HffX7 & 1 & $0.3 \%$ \\
(c.211_217dupCGCAGCA) & & \\
p.R50X (c.148C > T) / p.R576Q & 1 & $0.3 \%$ \\
p.R50X (c.148C > T) / p.L132WfsX153 (c.393delG) & 1 & $0.3 \%$ \\
p.R50X (c.148C > T) / p.Q176P (c.527A > C) & 1 & $0.3 \%$ \\
p.R491Afs (c.1470dupG) / p.R491Afs (c.1470dupG) & 1 & $0.3 \%$ \\
p.R94W (c.280C > T) / p.R94W (c.280C > T) & 1 & $0.3 \%$ \\
p.G135R (c.403G > A) / p.R70C & 1 & $0.3 \%$ \\
c.244-3_244-2delCA / c.1093-1G > T & 1 & $0.3 \%$ \\
p.R50X (c.148C > T) / p.H35R (c.104A > G) & 1 & $0.3 \%$ \\
p.W798R (c.2392 T > C) / p.A365V (c.1094C > T) & 1 & $0.3 \%$ \\
\hline
\end{tabular}

${ }^{a}$ Unidentified mutation in one allele

in the proportion of patients with fixed muscle weakness $(25 \% \rightarrow 21 \%)$ [8]. Thus, virtually all patients have exercise intolerance, which has been accompanied by repeated episodes of myoglobinuria (that patients typically refer to as 'dark urine') in half of the cohort. In addition, the vast majority of patients are able to report the second wind phenomenon, which they typically refer to as the ability to resume exercise with attenuated fatigue, tachycardia and myalgia after they take a quick rest. Importantly, this phenomenon was easily measurable in all patients (see Additional file 3 for a representative example), except in 1 child (aged 12 years), which also concurs with our previous observation that, as opposed to adults, the second wind may not be easily detectable in some of the youngest patients [20].

A laboratory feature that characterises the disease and can further assist in its diagnosis is the typically high levels of the muscle damage marker, serum CK activity, which is above reference limits in nearly all patients and above $2000 \mathrm{U} / \mathrm{L}$ in $\sim 2$ thirds of them, and is also in accordance with our previous report [8]. Other characteristics also remain essentially unchanged, such as frequency distribution among severity classes [at least when grouping the 2 highest severity classes (2 and 3 ) together], and with the same percentage (8\%) of patients who are virtually asymptomatic during normal daily living ( $i e$, belonging to class 0 , which denotes exercise intolerance only during strenuous activities or sports participation and absence of myoglobinuria episodes). Also, association with comorbidities induced directly or indirectly by the disease appears low, with very few cases of chronic renal failure and a prevalence of major noncommunicable diseases (diabetes, cardiovascular disease, cancer) that does not appear to differ from that expected for the adult Spanish population. Further, 1 patient has reached remarkable longevity
Table 2 Main phenotype data in all Spanish McArdle patients $(n=333)$

\begin{tabular}{|c|c|c|c|}
\hline & $\begin{array}{l}\mathrm{N} \text { with } \\
\text { data }\end{array}$ & $\begin{array}{l}\text { Result } \\
\text { (men + women) }\end{array}$ & $\begin{array}{l}\text { Main change with } \\
\text { regards to previous data } \\
(\mathrm{n}=239 \text { patients) }[1]\end{array}$ \\
\hline Gender & & $55 \%$ male & $\leftrightarrow$ \\
\hline $\begin{array}{l}\text { Age, years } \\
\text { (mean } \pm S D \text {, range) }\end{array}$ & 333 & $\begin{array}{l}48 \pm 19 \\
(12,99)^{a}\end{array}$ & $\uparrow$ \\
\hline $\begin{array}{l}\mathrm{BMI}, \mathrm{kg} / \mathrm{m}^{2} \\
\text { (mean } \pm \mathrm{SD} \text {, range) }\end{array}$ & 132 & $\begin{array}{l}24.7 \pm 4.8 \\
(16,43)\end{array}$ & $\leftrightarrow$ \\
\hline $\begin{array}{l}\text { Familial } \\
\text { consaguinity } \\
(\%)\end{array}$ & 188 & $17 \%$ & $\uparrow$ \\
\hline $\begin{array}{l}\text { Symptomatic } \\
\text { father }(\%)^{\mathrm{a}}\end{array}$ & 201 & $3 \%$ & $\leftrightarrow$ \\
\hline $\begin{array}{l}\text { Symptomatic } \\
\text { mother }(\%)^{a}\end{array}$ & 201 & $6 \%$ & $\leftrightarrow$ \\
\hline $\begin{array}{l}\text { Symptoms' } \\
\text { onset (\%) }\end{array}$ & 235 & & \\
\hline 1st decade & & $66 \%$ & $\uparrow$ \\
\hline 2nd decade & & $20 \%$ & $\downarrow$ \\
\hline 3rd decade & & $5 \%$ & $\leftrightarrow$ \\
\hline$\geq 4$ th decade & & $9 \%$ & $\leftrightarrow$ \\
\hline $\begin{array}{l}\text { Genetic } \\
\text { diagnosis (\%) }\end{array}$ & 275 & & \\
\hline 1st decade & & $5 \%$ & $\leftrightarrow$ \\
\hline 2nd decade & & $23 \%$ & $\leftrightarrow$ \\
\hline 3rd decade & & $23 \%$ & $\leftrightarrow$ \\
\hline$\geq 4$ th decade & & $49 \%$ & $\leftrightarrow$ \\
\hline $\begin{array}{l}\text { Exercise } \\
\text { intolerance (\%) }\end{array}$ & 272 & $99.6 \%$ & $\leftrightarrow$ \\
\hline $\begin{array}{l}\text { Second wind, } \\
\text { self-reported (\%) }\end{array}$ & 164 & $91.5 \%$ & $\uparrow$ \\
\hline $\begin{array}{l}\text { Second wind, } \\
\text { laboratory- } \\
\text { determined (\%) }\end{array}$ & 119 & $99.2 \%$ & $\leftrightarrow$ \\
\hline $\begin{array}{l}\text { Fixed muscle } \\
\text { weakness (\%) }\end{array}$ & 240 & $21 \%$ & $\downarrow$ \\
\hline $\begin{array}{l}\text { Recurrent episodes } \\
\text { of myoglobinuria } \\
\text { (\%) }\end{array}$ & 240 & $51 \%$ & $\leftrightarrow$ \\
\hline $\begin{array}{l}\text { Disease } \\
\text { severity }(\%)^{b}\end{array}$ & 240 & & \\
\hline Class 0 & & $8 \%$ & $\leftrightarrow$ \\
\hline Class 1 & & $41 \%$ & $\leftrightarrow$ \\
\hline Class 2 & & $30 \%$ & $\uparrow$ \\
\hline Class 3 & & $21 \%$ & $\downarrow$ \\
\hline $\begin{array}{l}\text { Disease } \\
\text { progression (\%) }\end{array}$ & 151 & & \\
\hline Improvement & & $34 \%$ & $\uparrow \uparrow$ \\
\hline Worsening & & $28 \%$ & $\leftrightarrow$ \\
\hline Constant & & $35 \%$ & $\downarrow \downarrow$ \\
\hline
\end{tabular}


Table 2 Main phenotype data in all Spanish McArdle patients $(\mathrm{n}=333)$ (Continued)

\begin{tabular}{|c|c|c|c|}
\hline & $\begin{array}{l}\mathrm{N} \text { with } \\
\text { data }\end{array}$ & $\begin{array}{l}\text { Result } \\
\text { (men + women) }\end{array}$ & $\begin{array}{l}\text { Main change with } \\
\text { regards to previous data } \\
\text { ( } \mathrm{n}=239 \text { patients) [1] }\end{array}$ \\
\hline $\begin{array}{l}\text { Acute renal } \\
\text { failure (\%) }\end{array}$ & 173 & $6 \%$ & $\leftrightarrow$ \\
\hline $\begin{array}{l}\text { Chronic renal } \\
\text { failure (\%) }\end{array}$ & 171 & $1 \%$ & $\leftrightarrow$ \\
\hline \multicolumn{4}{|l|}{ Comorbidities (\%) } \\
\hline Diabetes $^{c}$ & 140 & $9 \%$ & $\leftrightarrow$ \\
\hline CAD & 136 & $9 \%$ & $\leftrightarrow$ \\
\hline Hypertension & 135 & $11 \%$ & $\leftrightarrow$ \\
\hline Cancer & 134 & $1 \%$ & $\leftrightarrow$ \\
\hline Obesity & 127 & $10 \%$ & $\leftrightarrow$ \\
\hline COPD & 131 & $1 \%$ & $\leftrightarrow$ \\
\hline $\begin{array}{l}\text { Serum CK (\%) } \\
>200 \mathrm{U} / \mathrm{L} \\
>1000 \mathrm{U} / \mathrm{L}\end{array}$ & 179 & $98 \%, 68 \%$ & $\leftrightarrow, \downarrow$ \\
\hline $\begin{array}{l}\text { Biopsy } \\
\text { diagnosis (\%) }\end{array}$ & 205 & $100 \%$ & $\leftrightarrow$ \\
\hline $\begin{array}{l}\text { Physical activity } \\
\text { data (\% active) }\end{array}$ & 120 & $50 \%$ & $\uparrow \uparrow$ \\
\hline $\begin{array}{l}\mathrm{VO}_{2} \text { peak, } \mathrm{mLO}_{2} / \\
\mathrm{kg} / \mathrm{min} \\
(\text { mean } \pm \mathrm{SD} \text {, range })\end{array}$ & 120 & $\begin{array}{l}19.9 \pm 6.6 \\
(5.9,41.5)\end{array}$ & $\leftrightarrow$ \\
\hline \multicolumn{4}{|c|}{$\begin{array}{l}\text { Data on } 3 \text { patients (all males) who died recently (all after the 6th decade of } \\
\text { life, due to cardiovascular disease) are included } \\
\text { Symbols: ' 'symptomatic' refers to having the main symptomatic features of } \\
\text { McArdle disease (i.e., exercise intolerance with or without myoglobinuria or } \\
\text { self-reported second wind) } \\
\text { bdisease severity class following the classification originally reported by } \\
\text { Martinuzzi et al. [2]: } 0 \text { = asymptomatic or virtually asymptomatic (mild exercise } \\
\text { intolerance, but no functional limitation in any daily life activity); } 1 \text { = exercise } \\
\text { intolerance, contractures, myalgia, and limitation of acute strenuous exercise, } \\
\text { and occasionally in daily life activities; no record of myoglobinuria, no muscle } \\
\text { wasting or weakness; } 2 \text { = same as } 1 \text {, plus recurrent exertional myoglobinuria, } \\
\text { moderate restriction in exercise, and limitation in daily life activities; } 3 \text { = same } \\
\text { as } 2 \text {, plus fixed muscle weakness, with or without wasting and severe } \\
\text { limitations on exercise and most daily life activities } \\
\text { 'diabetes diagnosed based on a glucose tolerance test. Abbreviations: BMI, } \\
\text { body mass index; } C A D, \text { coronary artery disease; COPD, chronic obstructive } \\
\text { pulmonary disease; } \mathrm{VO}_{2} \text { peak, peak oxygen uptake }\end{array}$} \\
\hline
\end{tabular}

(99 years), supporting the overall benign nature of the disease compared with other glycogenoses.

An important problem that persists with regards to the first series [8] is that, despite most patients reporting onset of exercise intolerance symptoms since childhood, typically in physical education classes and in the school playground, genetic diagnosis has been delayed until much later in life. Among the 94 new patients, genetic diagnosis has not been available until the age of $20+$ years in $75 \%$ of cases, despite the vast majority (90\%) reporting symptoms before that age. Yet a major difference compared with our previous report is an increase in the proportion of patients (i) reporting a 4-year improvement in disease severity $(21 \% \rightarrow 34 \%)$ at the expense of those showing no change $(51 \% \rightarrow 35 \%)$; and (ii) adopting an active lifestyle in recent times $(32 \% \rightarrow 50 \%)$. Indeed, those patients who are physically active are 14-fold more likely to report an improvement after a 4-year period in the clinical course of the disease compared with their inactive peers (OR: 13.98; 95\%CI: 5.6, 34.9; $p<0.001)$. In addition, a key fitness and health indicator, $\mathrm{VO}_{2}$ peak, is significantly $(p=0.001)$ higher in physically active patients $(20.7 \pm 6.0)$ than in their inactive referents $(16.8 \pm 5.3 \mathrm{~mL} / \mathrm{kg} / \mathrm{min})$.

Age has a detrimental effect on several phenotypic features of the disease. The mean age ( $57 \pm 19$ years) of those patients in the highest severity class 3 (that includes presence of fixed muscle weakness) is higher than in those in the lower severity classes 1 (46 \pm 19 years, $p=0.007)$ and 2 (41 \pm 17 years, $p<0.001)$. Finally, PYGM genotype is not significantly associated with any of the phenotype data reported in Table 2 after controlling for sex and age (data not shown). Likewise, PYGM is not associated with any phenotype data within the subset of patients reporting a 4-year improvement in disease severity, with a similar proportion of PYGM alleles harbouring a missense mutation in this subgroup compared with the whole patient cohort (i.e., 30\% of total number of alleles in both cases).

\section{Discussion}

This is the largest series of patients' data that is available to date on McArdle disease and as such can provide corroborative or novel insights on this disorder. Despite heterogeneity, mainly in terms of PYGM genotype but also of disease severity [with almost 1 in 10 patients being fundamentally asymptomatic in daily living (ie, belonging to Martinuzzi's class 0) vs. 2 in 10 being clearly limited in daily life activities and having fixed muscle weakness (class 3)], several features of the disorder, mainly pertaining to phenotype and laboratory data, are common to the vast majority of patients. This should raise the suspicion of the presence of McArdle disease until genetic confirmation is achieved. These common features include intolerance to strenuous exercise coupled with the second wind phenomenon in almost all patients, as well as basal hyper-CK-emia.

The PYGM genotype data shown here are in overall agreement with previous reports on smaller Caucasian cohorts $[3,4,6,7,11,21-23]$ with p.R50X/p.R50X and p.R50X/p.W798R combinations accounting for $43 \%$ of all PYGM genotypes and p.R50X being by far the commonest pathogenic genetic variant. In addition, we have identified 7 novel mutations that must be added to the list of pathogenic variants causing McArdle disease [24]. That heterogeneity of PYGM genotypes does not account for heterogeneity in the clinical manifestation of the disease is in agreement with previous studies showing no genotypephenotype correlation $[4,6,22]$. In fact, in our series, biochemical analysis of muscle biopsies consistently showed null myophosphorylase activity and most reported PYGM mutations have functional consequences, with many 
actually resulting in no gene transcript levels owing to a protective intracellular mechanism, the so-called nonsensemediated decay, which degrades transcripts that contain premature termination codons [25]. By contrast, regular PA is likely the main modulator of the phenotype manifestation of the disease, which explains the heterogeneous presentation of the disorder among patients despite all having the same defect at the muscle molecular level, that is, complete inability to metabolize glycogen stores.

A promising novel result is that a growing number of Spanish patients (around one half of them) are now adopting a relatively active lifestyle, which we believe is especially important when considering the strong, positive association between regular PA and a favourable progression of clinical symptoms. These results support and extend the findings from our previous report [8]. This recent tendency reflects, at least in part, the fact that patients are now following our recommendations [notably, during educational talks given by us (AL, AS, GNG, TP) to the patients in each yearly meeting of the Spanish Association of Glycogenosis Patients] to perform low-moderate intensity PA regularly. Furthermore, $\mathrm{VO}_{2}$ peak was significantly higher in the physically active patients. Indeed, 8 physically active adult patients (vs. only 2 inactive adult patients) have a $\mathrm{VO}_{2 \text { peak }} \geq 8 \mathrm{METs}$ (where 1 MET = resting metabolic rate or $3.5 \mathrm{~mL} \mathrm{O} / \mathrm{kg} / \mathrm{min}$ for most humans). This is also an important finding because 8 METs is the minimum threshold for optimal health, above which the risk for cardiovascular mortality in adults is significantly reduced compared with lower values [26]. Our findings on PA also support previous studies showing the benefits of regular, low-moderate intensity exercise training (bicycling, brisk walking) during 8 [19] to 14 weeks to increase the $\mathrm{VO}_{2 \text { peak }}$ of McArdle patients [27].

We believe an important challenge identified by us here as well as previously [8] is the fact that diagnosis of the disease is usually delayed until adulthood despite symptoms frequently occurring since childhood. Misdiagnosis might also be as a potential cause of delayed diagnosis of McArdle disease [28]. This undoubtedly calls for a better characterisation and monitoring of the disease from childhood and adolescence. The possibility of diagnosing McArdle disease should be clearly mentioned in the specific educational training of paediatricians because early diagnosis would favour implementation of regular PA habits (with carbohydrate ingestion and gradual warm-up before strenuous activities) since early childhood (coupled with a diet rich in complex carbohydrates to ensure constant availability of glucose to working muscles) [29]. In this regard, all affected children report problems during physical education classes because the latter usually involve strenuous exercises, such as running, which is particularly painful and fatiguing for all of them compared to their healthy peers. Early diagnosis is particularly important when considering that practice of regular, low-moderate PA usually tracks from childhood to adolescence and from adolescence into adulthood, thereby laying the foundation for a healthy lifestyle over life [30]. As for diagnostic tools, we believe that objective assessment of the second wind followed by genetic analyses using blood samples (quick screening initially for the three most prevalent mutations, p.R50X, p.W798R and p.G205S, which account, alone or in combination, for $\sim 74 \%$ of all PYGM mutant alleles, and then, whenever needed, searching for further mutations by Sanger sequencing or NGS of the PYGM gene) is the most efficient strategy, with no need for performing unpleasant muscle biopsies unless it is a requirement to identify the genetic alteration or to validate the molecular consequences of novel mutations in the PYGM gene. Other tests that were traditionally implemented, such as electromyography recordings or the ischaemic forearm test are probably not useful anymore. A question that remains unanswered however is pregnancy outcomes in all women, especially which is the best way of delivery, whether vaginal or caesarean.

\section{Conclusions}

The reported prevalence of McArdle disease seems to grow considerably despite genetic diagnosis being frequently delayed until adulthood and onset of symptoms occurring since childhood. Thus, awareness of this disease and monitorisation is still insufficient, especially among paediatricians. Until a genetic cure is available (which is not predicted in the near future), the current epidemiologic data support that adoption of an active lifestyle is the best medicine for these patients.

\section{Additional files}

Additional file 1: Distribution of pathogenic mutations causing McArdle disease in Spanish patients by exon/intron. (PDF $24 \mathrm{~kb}$ )

Additional file 2: Frequency distribution of pathogenic PYGM mutations by exon. (PDF $11 \mathrm{~kb}$ )

Additional file 3: Example of second wind assessment in one adult patient. (PDF $253 \mathrm{~kb}$ )

\section{Acknowledgements}

We thank the Spanish Association of Glycogenosis for their help and their support; without their contribution this article would not be possible. We thank Dr. Kenneth McCreath for editorial support.

\section{Funding}

Publication of the article funded by Cátedra ASISA-UEM (2016/UEM41). The research of Alejandro Lucia, Tomàs Pinós, Joaquin Arenas, Gisela NogalesGadea and Miguel Angel Martín in the field of McArdle disease and muscle diseases is funded by the Fondo de Investigaciones Sanitarias ISCIII (FIS, grant numbers PI15/00558, PI12/00914, PI16/01492, PI13/00855 PI14/00903, PI15/ 01756 and PI15/00431) and cofinanced by FEDER. Gisela Nogales-Gadea is supported by a Miguel Servet research contract (ISCIII CD14/00032 and FEDER) and by AFM Telethon Trampoline Grant 21108. The funding bodies had no role in the design of the study and collection, analysis, and interpretation of data. Alfonsina Ballester-Lopez is funded by an Fl Agaur fellowship. Helios ParejaGaleano is supported by the Cátedra ASISA-UEM (2016/UEM41). 


\section{Availability of data and materials}

All data generated during this study is included in this published article.

\section{About this supplement}

This article has been published as part of BMC Genomics Volume 18 Supplement 8, 2017: Proceedings of the 34th FIMS World Sports Medicine Congress. The full contents of the supplement are available online at https://bmcgenomics.biomed central.com/articles/supplements/volume-18-supplement-8.

\section{Authors' contributions}

$\mathrm{AL}, \mathrm{AS}$ and $\mathrm{GN}-\mathrm{G}$ obtained patient data, analysed and interpreted the patient data and wrote the manuscript. ABE, IV, AGQ, PSL, IGC, SA, AB-L, TP, and MAM obtained and analysed genotype data of patients from Spain. GP-M, JC-C, HP-G, MP, ALA, JA and TP were in charge of data collection and analysis. All authors read and approved the final manuscript.

\section{Ethics approval and consent to participate}

All participants were informed of the purposes of that project and signed a written consent. This study was approved by the local institutional ethics committees (CEIC i + 12 14/357, PT13/0010/0022) and was in accordance with the Declaration of Helsinki for Human Research.

\section{Consent for publication}

Not applicable.

\section{Competing interests}

The authors declare that they have no competing interests.

\section{Publisher's Note}

Springer Nature remains neutral with regard to jurisdictional claims in published maps and institutional affiliations.

\begin{abstract}
Author details
${ }^{1}$ Universidad Pablo de Olavide, Sevilla, Spain. ${ }^{2}$ Instituto de Investigación Hospital 12 de Octubre (i+12), Madrid, Spain. ${ }^{3}$ Grup de Recerca en Malalties Neuromusculars i Neuropediatriques, Department of Neurosciences, Institut d'Investigació en Ciències de la Salut Germans Trias i Pujol, Universitat Autònoma de Barcelona, Camí de les Escoles, s/n 08916 (Barcelona), Badalona, Spain. ${ }^{4}$ Servicio de Pediatría, Hospital Universitari Germans Trias i Pujol, Badalona, Spain. ${ }^{5}$ Centre for Biomedical Network Research on Rare Diseases (CIBERER), Instituto de Salud Carlos III, Madrid, Spain. '́ Laboratorio de Enfermedades Mitocondriales y Neuromusculares, Hospital 12 de Octubre, Madrid, Spain. ${ }^{7}$ Rare Diseases and Pediatric Medicine Group, Galicia Sur Health Research Institute, Complexo Hospitalario Universitario de Vigo (CHUVI), SERGAS, Vigo, Spain. ${ }^{8}$ Servicio de Neurología, Hospital Universitari Germans Trias i Pujol, Badalona, Spain. ${ }^{9}$ Universidad Europea de Madrid, Madrid, Spain. ${ }^{10}$ Departament de Patologia Mitocondrial i Neuromuscular, Hospital Universitari Vall d'Hebron, Institut de Recerca (VHIR), Universitat Autónoma de Barcelona, Barcelona, Spain.
\end{abstract}

Published: 14 November 2017

\section{References}

1. Lucia A, Nogales-Gadea G, Perez M, Martin MA, Andreu AL, Arenas J. McArdle disease: what do neurologists need to know? Nat Clin Pract Neurol. 2008:4(10):568-77.

2. Vissing J, Haller RG. A diagnostic cycle test for McArdle's disease. Ann Neurol. 2003;54(4):539-42.

3. Bruno C, Cassandrini D, Martinuzzi A, Toscano A, Moggio M, Morandi L, Servidei $\mathrm{S}$, Mongini T, Angelini C, Musumeci O, et al. McArdle disease: the mutation spectrum of PYGM in a large Italian cohort. Hum Mutat. 2006;27(7):718.

4. Martin MA, Rubio JC, Buchbinder J, Fernandez-Hojas R, del Hoyo P, Teijeira S, Gamez J, Navarro C, Fernandez JM, Cabello A, et al. Molecular heterogeneity of myophosphorylase deficiency (McArdle's disease): a genotype-phenotype correlation study. Ann Neurol. 2001;50(5):574-81.

5. Martinuzzi A, Sartori E, Fanin M, Nascimbeni A, Valente L, Angelini C, Siciliano G, Mongini T, Tonin P, Tomelleri G, et al. Phenotype modulators in myophosphorylase deficiency. Ann Neurol. 2003;53(4):497-502.

6. Quinlivan R, Buckley J, James M, Twist A, Ball S, Duno M, Vissing J, Bruno C, Cassandrini D, Roberts M, et al. McArdle disease: a clinical review. J Neurol Neurosurg Psychiatry. 2010;81(11):1182-8.
7. Vieitez I, Teijeira S, Fernandez JM, San Millan B, Miranda S, Ortolano S, Louis S, Laforet P, Navarro C. Molecular and clinical study of McArdle's disease in a cohort of 123 European patients. Identification of 20 novel mutations. Neuromuscul Disord. 2011;21(12):817-23.

8. Lucia A, Ruiz JR, Santalla A, Nogales-Gadea G, Rubio JC, Garcia-Consuegra I, Cabello A, Perez M, Teijeira S, Vieitez I, et al. Genotypic and phenotypic features of McArdle disease: insights from the Spanish national registry. J Neurol Neurosurg Psychiatry. 2012;83(3):322-8.

9. De Castro M, Johnston J, Biesecker L. Determining the prevalence of McArdle disease from gene frequency by analysis of next-generation sequencing data. Genet Med. 2015;17(12):1002-6.

10. Haller RG. Treatment of McArdle disease. Arch Neurol. 2000;57(7):923-4.

11. Rubio JC, Garcia-Consuegra I, Nogales-Gadea G, Blazquez A, Cabello A, Lucia A, Andreu AL, Arenas J, Martin MA. A proposed molecular diagnostic flowchart for myophosphorylase deficiency (McArdle disease) in blood samples from Spanish patients. Hum Mutat. 2007;28(2):203-4.

12. Kubisch C, Wicklein EM, Jentsch TJ. Molecular diagnosis of McArdle disease: revised genomic structure of the myophosphorylase gene and identification of a novel mutation. Hum Mutat. 1998;12(1):27-32.

13. Garcia-Consuegra I, Rubio JC, Nogales-Gadea G, Bautista J, Jimenez S, Cabello A, Lucia A, Andreu AL, Arenas J, Martin MA. Novel mutations in patients with McArdle disease by analysis of skeletal muscle mRNA. J Med Genet. 2009;46(3):198-202.

14. Garcia-Consuegra I, Blazquez A, Rubio JC, Arenas J, Ballester-Lopez A, Gonzalez-Quintana A, Andreu AL, Pinos T, Coll-Canti J, Lucia A, et al. Taking advantage of an old concept, "illegitimate transcription", for a proposed novel method of genetic diagnosis of McArdle disease. Genet Med. 2016;18(11):1128-35.

15. Dimauro S, Tsujino S. Nonlysosomal glycogenoses. In: Engel AG, FranziniArmstrong C, editors. Myology. New York: McGraw-Hill; 1995. p. 1554-6.

16. Nadaj-Pakleza AA, Vincitorio CM, Laforet P, Eymard B, Dion E, Teijeira S, Vietez I, Jeanpierre M, Navarro C, Stojkovic T. Permanent muscle weakness in McArdle disease. Muscle Nerve. 2009;40(3):350-7.

17. WHO. Global recommendations on physical activity for health. In: Switzerland; 2010.

18. Physical activity guidelines for Americans. The Oklahoma nurse. 2008;53(4):25.

19. Mate-Munoz JL, Moran M, Perez M, Chamorro-Vina C, Gomez-Gallego F, Santiago C, Chicharro L, Foster C, Nogales-Gadea G, Rubio JC, et al. Favorable responses to acute and chronic exercise in McArdle patients. Clin J Sport Med. 2007;17(4):297-303.

20. Perez M, Ruiz JR, Fernandez Del Valle M, Nogales-Gadea G, Andreu AL, Arenas J, Lucia A. The second wind phenomenon in very young McArdle's patients. Neuromuscul Disord. 2009;19(6):403-5.

21. Aquaron R, Berge-Lefranc JL, Pellissier JF, Montfort MF, Mayan M, FigarellaBranger D, Coquet M, Serratrice G, Pouget J. Molecular characterization of myophosphorylase deficiency (McArdle disease) in 34 patients from southern France: identification of 10 new mutations. Absence of genotype-phenotype correlation. Neuromuscul Disord. 2007;17(3):235-41.

22. Deschauer M, Morgenroth A, Joshi PR, Glaser D, Chinnery PF, Aasly J, Schreiber H, Knape M, Zierz S, Vorgerd M. Analysis of spectrum and frequencies of mutations in McArdle disease. Identification of 13 novel mutations. J Neurol. 2007;254(6):797-802.

23. Rubio JC, Gomez-Gallego F, Santiago C, Garcia-Consuegra I, Perez M, Barriopedro MI, Andreu AL, Martin MA, Arenas J, Lucia A. Genotype modulators of clinical severity in McArdle disease. Neurosci Lett. 2007;422(3):217-22.

24. Nogales-Gadea G, Brull A, Santalla A, Andreu AL, Arenas J, Martin MA, Lucia A, de Luna N, Pinos T. McArdle disease: update of reported mutations and polymorphisms in the PYGM gene. Hum Mutat. 2015; 36(7):669-78.

25. Nogales-Gadea G, Rubio JC, Fernandez-Cadenas I, Garcia-Consuegra I, Lucia A, Cabello A, Garcia-Arumi E, Arenas J, Andreu AL, Martin MA. Expression of the muscle glycogen phosphorylase gene in patients with McArdle disease: the role of nonsense-mediated mRNA decay. Hum Mutat. 2008;29(2):277-83.

26. Kodama S, Saito K, Tanaka S, Maki M, Yachi Y, Asumi M, Sugawara A, Totsuka K, Shimano H, Ohashi Y, et al. Cardiorespiratory fitness as a quantitative predictor of all-cause mortality and cardiovascular events in healthy men and women: a meta-analysis. JAMA. 2009;301(19):2024-35.

27. Haller $R$, Wyrick $P$, Taivassalo $T$, Vissing J. Aerobic conditioning: an effective therapy in McArdle's disease. Ann Neurol. 2006;59(6):922-8. 
28. Scalco RS, Chatfield S, Junejo MH, Booth S, Pattni J, Godfrey R, Quinlivan R, McArdle Disease Misdiagnosed as Meningitis. Am J Case Rep. 2016;17:905-8.

29. Perez M, Foster C, Gonzalez-Freire M, Arenas J, Lucia A. One-year follow-up in a child with McArdle disease: exercise is medicine. Pediatr Neurol. 2008; 38(2):133-6.

30. Malina RM. Tracking of physical activity and physical fitness across the lifespan. Res Q Exerc Sport. 1996;67(3 Suppl):S48-57.

Submit your next manuscript to BioMed Central and we will help you at every step:

- We accept pre-submission inquiries

- Our selector tool helps you to find the most relevant journal

- We provide round the clock customer support

- Convenient online submission

- Thorough peer review

- Inclusion in PubMed and all major indexing services

- Maximum visibility for your research

Submit your manuscript at www.biomedcentral.com/submit
Biomed Central 